\title{
Oriented Gradient Maps based Automatic Asymmetric 3D-2D Face Recognition
}

\author{
Di Huang ${ }^{1,2}$, Mohsen Ardabilian ${ }^{2}$, Yunhong Wang ${ }^{1}$, Liming Chen $^{2}$ \\ ${ }^{1}$ Laboratory of Intelligent Recognition and Image Processing, Beijing Key Laboratory of Digital Media \\ School of Computer Science and Engineering, Beihang University, Beijing, 100191, China \\ ${ }^{2}$ Université de Lyon, CNRS, Ecole Centrale de Lyon, LIRIS, UMR5205, Lyon, 69134, France \\ \{di.huang, mohsen.ardabilian, liming.chen\}@ec-lyon.fr; yhwang@buaa.edu.cn
}

\begin{abstract}
Recently, asymmetric 3D-2D face recognition has been paid increasing attention. It enrolls in textured $3 D$ faces and performs identification using only $2 D$ facial images, therefore it generally achieves a better result than $2 D$ algorithms do, and avoids inconvenience of data acquisition and computation of $3 D$ methods as well. In this paper, a biological vision-based facial representation, namely Oriented Gradient Maps (OGMs), is introduced for such an application. It simulates the response of complex neurons to gradient information within a pre-defined neighborhood, and thus can describe local texture changes of $2 D$ faces and local geometry variations of $3 D$ faces at the same time. Due to its property of being highly distinctive, these OGMs improve accuracies of both matching steps of asymmetric face recognition, i.e. (1) $3 D-2 D$ matching using Canonical Correlation Analysis (CCA); (2) 2D-2D matching using LBP histogram based features and Sparse Representation Classifier (SRC). Some comparative experiments are carried out on the complete FRGC v2.0 database, and the achieved results clearly highlight the effectiveness of the biological vision-based facial description and its successful application to asymmetric face recognition.
\end{abstract}

\section{Introduction}

Face recognition is a critical and popular topic in the area of computer vision and pattern recognition for its wide application potential and scientific challenges. However, despite the great progress made in this domain [23], as a typical biometric feature, 2D facial images do not remain reliable when affected by changes of illumination, pose, facial expression, etc. Recently, 3D face recognition has emerged as a major alternative to deal with these unsolved issues in 2D domain, i.e. lighting and pose variations $[19,4]$. Unfortunately, 3D based approaches are currently limited by their acquisition, registration and computation cost.

More recently, asymmetric 3D-2D face recognition [18,
$8,9,17,22]$ has been attracting increasing interests, since it is expected to limit the use of 3D data where it really helps to improve face recognition accuracy. Unlike the traditional 2D, 3D and multi-modal face recognition approaches that require gallery and probe face data to own similar properties: 2D/3D, color/gray, or even to be captured by the same type of camera sensors, asymmetric 3D-2D face recognition methods assume that the gallery set consists of 3D face data, whilst the probe set only contains 2D facial images, which makes them more likely to be used in real-time environment than 3D based ones.

In contrast to a large number of investigations and contributions made within the field of $3 \mathrm{D}$ and textured $3 \mathrm{D}$ face recognition $[14,6,2,16,3,13,10,11,12,20]$, to the best of our knowledge, so far only a few tasks in the literature have addressed the problem on asymmetric 3D-2D face recognition. Rama et al. [17] presented the Partial Principle Component Analysis $\left(\mathrm{P}^{2} \mathrm{CA}\right)$ for feature extraction and dimensionality reduction on both the cylindrical texture representation (3D) in the gallery and 2D images in the probe. However, their 3D face data still only conveyed texture information rather than shape clues. In [18], Riccio et al. employed several control points to compute geometrical invariants for 2D/3D face recognition. Nevertheless, it brought in another difficulty in accurately locating these fiducial points on both the 2D and 3D facial data. Yang et al. [22] implemented a patch based Kernel CCA to learn the mapping between facial range and texture images in the gallery and probe sets respectively. But their gallery set only contained shape information, and the original intensity and depth information (pixel values in the facial range and texture image) cannot comprehensively describe variations of facial appearances. Furthermore, all the tasks above partially relied on 2D faces, and none of them provided reliable performance when lighting condition or pose status changes.

In the previous study [8], we introduced to enroll in textured 3D face models, each of which includes a depth face map and its corresponding 2D texture counterpart, and the resulting framework of asymmetric 3D-2D face recognition 
was thereby composed of two separate matching steps, i.e. 2D-2D and 3D-2D face matching. In the former step, SRC [21] was exploited to LBP histogram based facial features, while in the latter one, CCA was utilized to learn the mapping between facial range and texture images in LBP feature space. Both the similarity measures were combined to make the final decision. In addition, we designed a preprocessing pipeline [9] to improve its robustness to lighting and moderate pose changes. This asymmetric 3D-2D face recognition method achieved better results than 2D image based ones while keeping the computational cost under control. Taking into account its advantages, we follow this framework.

This paper presents a new biological vision-based facial description, namely Oriented Gradient Maps (OGMs). The OGMs simulate the response of complex neurons to gradient information within a given neighborhood, and are able to describe local texture changes of $2 \mathrm{D}$ facial maps and local shape changes of 3D facial maps at the same time. Due to its property of being highly distinctive, the OGMs improve the performance of both face matching steps of the asymmetric 3D-2D face recognition. Specifically, in 3D-2D matching, these OGMs of 2D and 3D facial images are used to replace 2D and 3D LBP faces as the input of CCA, while in 2D2D matching, instead of extracting LBP histograms directly from original 2D facial images, a set of OGMs are generated as distinctiveness enhanced intermediate facial descriptions, their LBP histogram-based features are then fed into SRC. Finally, the holistic 3D-2D matching score of CCA is fused with that of feature-based 2D-2D matching for identification. Moreover, almost all the previous approaches for asymmetric 3D-2D face recognition were evaluated either on small private datasets or subsets of public ones; as a result, it is difficult to compare the performance and test their robustness as a comprehensive database is utilized. In contrast, all the experiments designed in this paper are carried out on the complete FRGC v2.0 database, and the results clearly illustrate the effectiveness of the biological visionbased facial representation as well as its successful application to asymmetric 3D-2D face recognition.

The remainder of this paper is organized as follows: the proposed biological vision-based facial representation is introduced in section 2, and section 3 presents the asymmetric 3D-2D face recognition approach. Experimental results are described and analyzed in section 4 . Section 5 concludes the paper.

\section{Oriented Gradient Maps}

To increase the distinctiveness of human faces, we introduce in this section a biological vision-based facial representation, which can be applied to describe both range and texture facial images.

This facial description is inspired by the study of Edelman et al. [5], who proposed such a conception of the com- plex neuron in primary visual cortex. The complex neurons respond to a gradient at a particular orientation and spatial frequency, but the location of the gradient is allowed to shift over a small receptive field rather than being precisely localized. Recently, it has been successfully exploited for multimodal face recognition in [11] and has proved its discriminative power to represent both types of face data, i.e. facial range and texture images. In this work, we further investigate it in asymmetric 3D-2D face recognition, which makes use of these two types of data but in a different manner.

\subsection{Representation of the complex neuron response}

The proposed facial representation aims to simulate the response of complex neurons. It is based on a convolution of gradients in specific directions in a given circular neighborhood, and its radius value can be changed. The precise radius value $R$ of the circular neighborhood region needs to be fixed experimentally for different applications.

Specifically, the response of a complex neuron at a given pixel location $(x, y)$ is its gradient map of every orientation convolved with a Gaussian kernel. Given a raw input image $I$, a certain number of gradient maps $G_{1}, G_{2}, \ldots, G_{o}$, one for each quantized direction $o$, are first computed. They are formally defined as:

$$
G_{o}=\left(\frac{\partial I}{\partial o}\right)^{+}
$$

where the ' + ' sign means that only positive values are kept to preserve the polarity of the intensity changes, while the negative ones are set to zero.

Each gradient map describes gradient norms of the input image in a direction $o$ at every pixel location. We then simulate the response of complex neurons by convolving its gradient maps with a Gaussian kernel $G$. The standard deviation of the Gaussian kernel $G$ is proportional to the radius of the given neighborhood area, $R$, as in (2).

$$
\rho_{o}^{R}=G_{R} * G_{o}
$$

The purpose of the convolution with Gaussian kernels is to allow the gradients to shift within a neighborhood without abrupt changes.

At a given pixel location $(x, y)$, we collect all the values of the convolved gradient maps at that location and build the vector $\rho^{R}(x, y)$ thus having a response value of complex neurons.

$$
\rho^{R}(x, y)=\left[\rho_{1}^{R}(x, y), \cdots, \rho_{O}^{R}(x, y)\right]^{t}
$$

This vector, $\rho^{R}(x, y)$, is further normalized to unit norm vector, which is called in the subsequent response vector and denoted by $\rho^{R}$. 


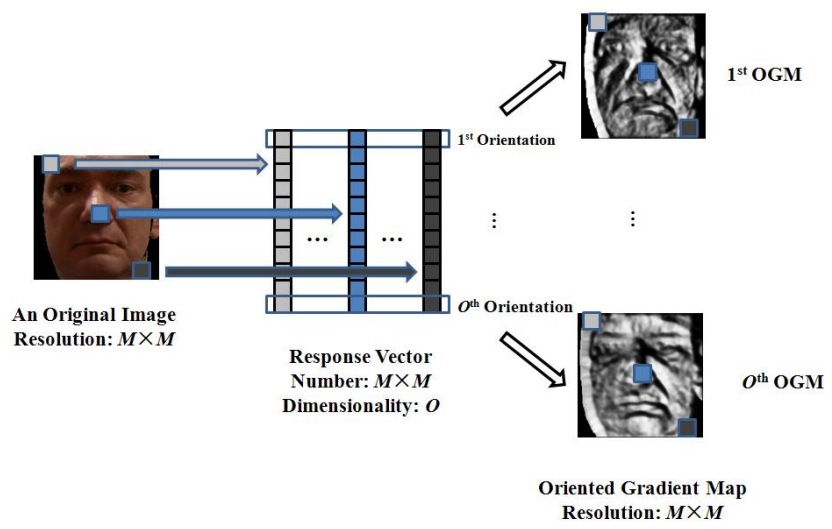

Figure 1. An illustration of the oriented gradient maps for each of the quantized orientations $o$.

\subsection{Facial description by response vectors}

Now both facial range and texture images can be represented by the values of complex neurons according to the response vectors. Specifically, given a facial range or texture image $I$, we generate an Oriented Gradient Map (OGM) $J_{o}$ using complex neurons for each orientation $o$ defined as:

$$
J_{o}(x, y)=\underline{\rho}_{o}^{R}(x, y)
$$

Figure 1 illustrates such a process. In our work, we generate eight OGMs for eight quantized directions. Instead of the original facial range and texture images, the OGMs are further fed to the following steps for face matching.

\subsection{The properties of distinctness and invariance}

The proposed OGM based facial description potentially offers high distinctiveness because it highlights the details of local shape and texture changes. See Fig. 2 for some examples. Meanwhile, it also possesses the property of being robustness to affine lighting and shape transformations.

When applied to 2D facial texture images, the proposed OGMs display the property of being robust to affine lighting transformations. Indeed, an OGM $J_{o}$ is simply the normalized convolved facial gradient map at orientation $o$ according to (4), while a brightness change usually adds a constant intensity value, so it does not affect the gradient computation. Furthermore, a change in image contrast in which the intensities of all the pixels are multiplied by a constant will result in the multiplication of gradient calculation; however, this change of contrast will be cancelled by the normalization of the response vector. Similarly, the OGMs of facial range images are also invariant to affine geometric transformations.

The proposed OGMs can be made even rotation invariant if we choose to quantize directions starting from the principal direction of all gradients within the given neighborhood. But we do not perform such rotation normalization for sav-

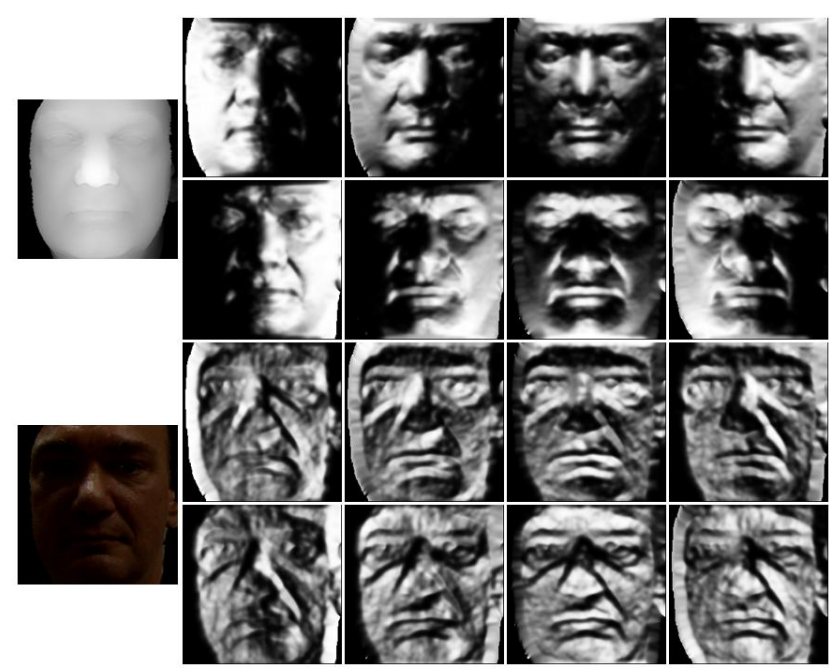

Figure 2. The eight OGMs of a range face and its corresponding texture.

ing computational cost as face data are generally in an upright frontal position in user cooperative applications.

\section{Asymmetric 3D-2D Face Recognition}

Recall that we follow the asymmetric 3D-2D face recognition framework proposed in $[8,9]$, which makes uses of two types of face data in the gallery set, i.e. facial range and texture images; while only 2D facial images are regarded as probes. Therefore, two independent face matching steps are contained: 3D-2D and 2D-2D. After facial representation, each range or texture face is described by its OGMs, denoted as $\left\{J_{R 1}, J_{R 2}, \ldots, J_{R o}\right\}$ or $\left\{J_{T 1}, J_{T 2}, \ldots, J_{T o}\right\}$; where $o$ is the number of quantized orientations.

\subsection{D-2D Face Matching (ASY)}

In this step, probe 2D faces are matched with the range faces in the gallery set. For a probe $2 \mathrm{D}$ facial image $P_{T}$, its similarity with a gallery range face $G_{T}$ is calculated by first computing the score $S_{i}^{A s y}$ between each OGM pair of certain quantized direction: $J_{T i}^{P}$ and $J_{R i}^{G}, i=\{1,2, \ldots, o\}$, independently, and then combining them with a fusion scheme.

CCA [7] is a powerful analysis method especially useful for relating two sets of variables, by maximizing correlation in the CCA subspace. In this paper, it is introduced to learn the mapping between each range and texture OGM pair $\left(J_{T i}\right.$ and $J_{R i}$ ).

Given $N$ pairs of samples $\left(x_{i}, y_{i}\right)$ of $(X, Y), i=1,2, \ldots$, $N$, where $X \in J_{T}, Y \in J_{R}$, with the mean value of zero. The goal of CCA is to learn a pair of directions $w_{x}$ and $w_{y}$ to maximize correlation between $x=w_{x}^{T} X$ and $y=w_{y}^{T} Y$. In the context of CCA, the two projections: $x$ and $y$ are also referred as canonical variants. Formally, the two directions 
can be calculated as the maxima of the function:

$$
\rho=\frac{E\left[w_{x}^{T} X Y^{T} w_{y}\right]}{\sqrt{E\left[w_{x}^{T} X X^{T} w_{x}\right] E\left[w_{y}^{T} Y Y^{T} w_{y}\right]}}
$$

To test new pairs of variables, we first project them into CCA subspace $x^{\prime}=w_{x}^{T} X^{\prime} ; y^{\prime}=w_{y}^{T} Y^{\prime}$; and then their similarity is computed by (6), where a bigger value indicates a higher similarity.

$$
S\left(x^{\prime}, y^{\prime}\right)=\frac{x^{\prime} \cdot y^{\prime}}{\left\|x^{\prime}\right\|\left\|y^{\prime}\right\|}
$$

\subsection{D-2D Face Matching (SYM)}

In this step, 2D face images in the probe set are matched with the ones in the gallery set. Considering that the similarity measurement of 3D-2D matching and the one of 2D2D matching will be further combined for the final decision, the matching scores of both parts are expected to be complementary to each other. Since the score achieved in 3D-2D face matching is calculated in a holistic way, in this subsection, we exploit a feature-based approach, LBP, for face matching.

LBP is employed in the same manner as in [1], by firstly dividing the whole face into several sub-regions from which LBP based histograms are extracted; then combining all the local histograms to form a global one as the final facial representation. Unlike [1], LBP is not applied to original 2D facial images, but on its OGMs instead, because OGMs improve their distinctiveness. Furthermore, we use SRC [21] to compute similarity scores between LBP histogram based features of two faces, since it has proved that SRC is much more effective than Chi-square distance for classification of histogram based features [9].

Similarly, the score $S_{i}^{\text {Sym }}$ is calculated based on LBP features of $J_{T i}^{P}$ and $J_{T i}^{G}, i=\{1,2, \ldots, o\}$, using SRC independently; then they are combined with a fusion scheme.

\subsection{Score Fusion}

Because the proposed biological vision-based facial description generates an OGM for each quantized gradient orientation, either for 2D-2D or 3D-2D face matching, the similarity measurement $F S^{S y m}$ or $F S^{A s y}$ combines the ones of all $o$ OGMs ( $S_{i}^{\text {Sym }}$ or $S_{i}^{A s y}$ ). The final similarity score of asymmetric 3D-2D face recognition, $F$, is achieved by further fusing $F S^{S y m}$ or $F S^{A s y}$.

All fusion steps in this paper are computed according to a simple sum rule to highlight the effectiveness of this asymmetric face recognition framework, and more powerful fusion strategies can be explored for a further improvement. Before that, all the scores are normalized to the interval of $[0,1]$ by using min-max normalization and different polarities should be reversed.

\section{Experimental Results}

The experiments were carried out on the complete FRGC v2.0 database [15]. It is one of the most comprehensive and popular databases, made up of 4007 textured 3D face models of 466 subjects. The preprocessing pipeline [9], consisting of Logarithmic Total variation (LTV) based illumination normalization and Active Appearance Model (AAM) based pose correction, was exploited. 11 out of 64 landmarks provided by AAM were selected to register 3D face models in the gallery set. All the facial range and texture images were converted to the pre-defined mean shape. The first 3D face model with a neutral expression from each subject formed a gallery set of 466 samples. The remaining texture faces $(4007-466=3541)$ were treated as probes. All the facial images were further cropped to $175 \times 190$ pixels as input of the subsequent experiments.

We designed three experiments: the first one is to evaluate the proposed facial representation both in 2D-2D and 3D-2D face matching; the second is to analyze the impact of the neighborhood area radius $R$ of OGMs on each matching step; and the last is to show the result of the asymmetric $3 \mathrm{D}-2 \mathrm{D}$ face recognition when combining the similarity scores of both separate face matching steps.

\subsection{Performance based on OGMs}

In this sub-section, we executed both 2D-2D and 3D-2D face matching on the OGMs of facial range and texture images to show their efficiency. Recall that, each facial range or texture image was already represented by a set of OGMs; therefore, as described in Section 3, both the 2D-2D and $3 \mathrm{D}-2 \mathrm{D}$ face matching steps were applied on the OGMs of facial range and texture images instead of the original ones. To calculate OGMs, we set the radius value of OGMs at 1 . In the LBP operator, we used uniform pattern, and the number of sampling points was set to 8 while the distance between the central pixel and its neighboring points was fixed at $2\left(L B P_{(8,2)}^{U 2}\right)$ as did in [8].

Table 1 demonstrates that in 2D-2D face matching, using each OGM of the original 2D facial images (after preprocessing pipeline), PCA based features achieve better results than LBP based ones do. However, when combining these similarity scores of all OGMs to calculate a final accuracy, LBP slightly outperforms PCA. To both types of features, i.e. PCA and LBP based ones, operating on each OGM and then fusing all the similarity measurements generates much better performance than directly applying them on original 2D faces, which indicates that the proposed OGMs improve the distinctiveness of original 2D facial images, leading to higher performance in 2D-2D matching.

In 3D-2D matching, as illustrated in Table 2, the result based on $L B P_{(8,2)}^{U 2}$ is not as good as that of original faces, which contradicts the conclusion in [8]. The reason is probably that the database used in [8] was only a small subset of 


\begin{tabular}{l|c}
\hline \hline 2D-2D Matching & Accuracy \\
\hline \hline (Sym01) OGM1 + LBP Histograms & 0.8114 \\
\hline (Sym02) OGM2 + LBP Histograms & 0.8082 \\
\hline (Sym03) OGM3 + LBP Histograms & 0.8018 \\
\hline (Sym04) OGM4 + LBP Histograms & 0.8116 \\
\hline (Sym05) OGM5 + LBP Histograms & 0.8068 \\
\hline (Sym06) OGM6 + LBP Histograms & 0.8150 \\
\hline (Sym07) OGM7 + LBP Histograms & 0.8130 \\
\hline (Sym08) OGM8 + LBP Histograms & 0.8170 \\
\hline (Sym09) OGM1 + PCA & 0.8879 \\
\hline (Sym10) OGM2 + PCA & 0.8924 \\
\hline (Sym11) OGM3 + PCA & 0.8834 \\
\hline (Sym12) OGM4 + PCA & 0.8743 \\
\hline (Sym13) OGM5 + PCA & 0.8772 \\
\hline (Sym14) OGM6 + PCA & 0.8848 \\
\hline (Sym15) OGM7 + PCA & 0.8577 \\
\hline (Sym16) OGM8 + PCA & 0.8786 \\
\hline (Sym17) OGMs (Sym01-08) + LBP Histograms & $\mathbf{0 . 9 3 9 0}$ \\
\hline (Sym18) OGMs (Sym09-16) + PCA & 0.9365 \\
\hline (Sym19) Original Face + LBP Histograms & 0.7995 \\
\hline (Sym20) Original Face + PCA & 0.7854 \\
\hline \hline
\end{tabular}

Table 1 . The results of 2D-2D face matching based on different facial features using SRC.

\begin{tabular}{l|c}
\hline \hline 3D-2D Matching & Accuracy \\
\hline \hline (Asy01) OGM1 + PCA & 0.8772 \\
\hline (Asy02) OGM2 + PCA & 0.8726 \\
\hline (Asy03) OGM3 + PCA & 0.8582 \\
\hline (Asy04) OGM4 + PCA & 0.8644 \\
\hline (Asy05) OGM5 + PCA & 0.8630 \\
\hline (Asy06) OGM6 + PCA & 0.8690 \\
\hline (Asy07) OGM7 + PCA & 0.8421 \\
\hline (Asy08) OGM8 + PCA & 0.8582 \\
\hline (Asy09) $L B P_{(8,1)}^{U 2}$ Image + PCA & 0.6461 \\
\hline (Asy10) $L B P_{(8,2)}^{U 2}$ Image + PCA & 0.7636 \\
\hline (Asy11) $L B P_{(8,3)}^{U 2}$ Image + PCA & 0.8128 \\
\hline (Asy12) $L B P_{(8,4)}^{U 2}$ Image + PCA & 0.8328 \\
\hline (Asy13) $L B P_{(8,5)}^{U 2}$ Image + PCA & 0.8492 \\
\hline (Asy14) $L B P_{(8,6)}^{U 2}$ Image + PCA & 0.8585 \\
\hline (Asy15) $L B P_{(8,7)}^{U 2}$ Image + PCA & 0.8540 \\
\hline (Asy16) $L B P_{(8,8)}^{U 2}$ Image + PCA & 0.8571 \\
\hline (Asy17) OGMs (Asy01-Asy08) & $\mathbf{0 . 9 4 0 4}$ \\
\hline (Asy18) MS-LBP (Asy09-Asy16) & 0.8700 \\
\hline (Asy19) Original Face + PCA & 0.8170 \\
\hline \hline
\end{tabular}

Table 2. The results of 3D-2D face matching based on different facial features using CCA.

FRGC v2.0. We further varied the radius value of the LBP operator from 1 to 8 pixels to observe the changes of perfor-

\begin{tabular}{l|c}
\hline \hline Different Fusion & Recognition Rate \\
\hline \hline (F01): (Sym17) + (Asy17) & $\mathbf{0 . 9 5 3 7}$ \\
\hline (F02): (Sym17) + (Asy18) & 0.9274 \\
\hline (F03): (Sym17) + (Asy19) & 0.9325 \\
\hline (F04): (Sym18) + (Asy17) & 0.9396 \\
\hline (F05): (Sym18) + (Asy18) & 0.9192 \\
\hline (F06): (Sym18) + (Asy19) & 0.9291 \\
\hline (F07): (Sym19) + (Asy17) & 0.9297 \\
\hline (F08): (Sym19) + (Asy18) & 0.8769 \\
\hline (F09): (Sym19) + (Asy19) & 0.8128 \\
\hline (F10): (Sym20) + (Asy17) & 0.9359 \\
\hline (F11): (Sym20) + (Asy18) & 0.9017 \\
\hline (F12): (Sym20) + (Asy19) & 0.8901 \\
\hline \hline
\end{tabular}

Table 3. Final results of the asymmetric 3D-2D face recognition by combining $2 \mathrm{D}-2 \mathrm{D}$ and $3 \mathrm{D}-2 \mathrm{D}$ matching steps.

mance, and we can see that when the radius value is larger than 3, LBP based accuracies are better than original face based one. Because multi-scale LBP (MS-LBP) is regarded as an effective solution for improved performance, all these results based on single LBP operators (Asy09-Asy16) are finally combined, and the resulted performance (Asy18) is indeed much better than any of the single LBP based one. On the other hand, the OGM based recognition rate by fusing similarity scores of its eight directions (Asy01-Asy08) is up to $94 \%$, which is much better than that based on original faces (Asy19), any single LBP faces (Asy09-Asy16), and MS-LBP (Asy18). The great improvement on recognition rate suggests that the proposed OGM based facial representation is more effective than LBP for 3D-2D matching.

\subsection{Radius analysis of OGM neighborhood}

Recall that complex neurons respond to gradient information within a neighborhood which is defined as a circular region in our implementation. In our experiments, we evaluated different values of radius $R$ and studied its impacts on the performance of both $2 \mathrm{D}-2 \mathrm{D}$ and $3 \mathrm{D}-2 \mathrm{D}$ face matching. In 2D-2D matching, the operator, $L B P_{(8,2)}^{U 2}$, was utilized.

From Fig. 3, it can be seen that in both the 2D-2D and 3D-2D face matching, when we increase the radius value of OGM neighborhood, the performance of each OGM and their fusion degrades. The best results are achieved as the radius value is set at 1 in both the matching steps.

\subsection{Final combination of two face matching steps}

To achieve the final performance of asymmetric 3D-2D face recognition, both similarity scores of the two separate matching steps, i.e., 2D-2D and 3D-2D face matching, were fused. The results of different combinations are compared in Table 3.

The last four rows (Sym17-Sym20) of Table 1 for 2D-2D matching, and the last three rows (Asy17-Asy19) of Table 2 


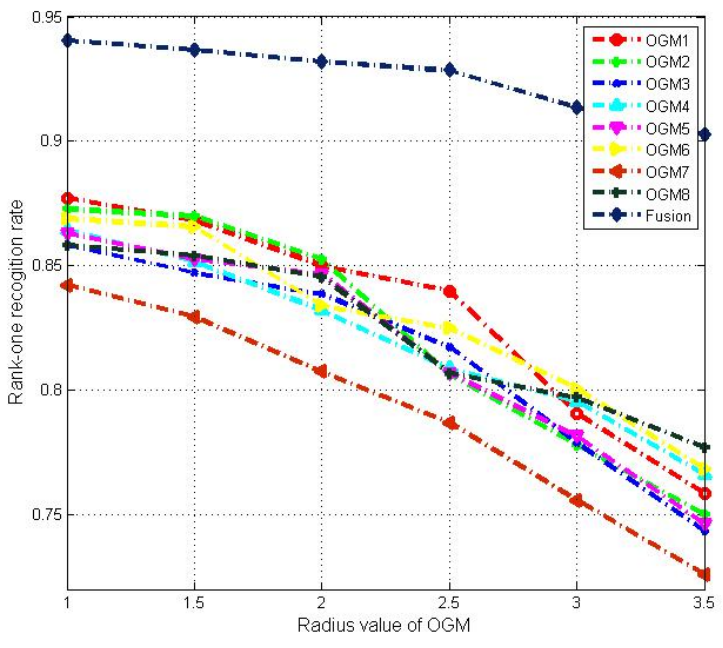

(a)

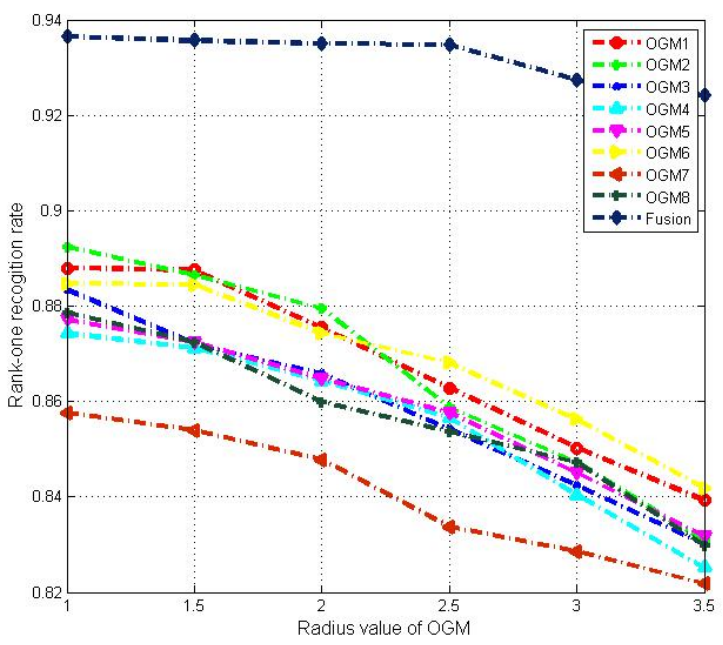

(b)

Figure 3. The performance curves based on different radii of each OGM as well as their fusion accuracy: (a) 2D-2D matching; (b) 3D-2D matching.

for 3D-2D matching are utilized for final fusion. Comparing all possible combinations of the similarity measurements in two steps respectively, the best performance is achieved by fusing (Sym17) and (Asy17). Specifically, the performance of (Sym17) is based on LBP histogram based facial features extracted from OGMs for 2D-2D matching, and (Asy17) is computed by CCA using the OGMs of facial range and texture images. It proves once again that the OGM based facial representation is very effective to improve the distinctiveness of original texture or depth faces. Moreover, two different types of facial features in two face matching steps, i.e. (1) local ones, LBP histograms in 2D-2D matching and (2) holistic ones, PCA in 3D -2D matching are complementary to each other, and their fusion result is better than either of them.

According to the achieved experimental results, the proposed OGM-based method is competent in representing $2 \mathrm{D}$ and 3D faces, leading to satisfying accuracies in both 2D2D and 3D-2D matching. Meanwhile, the rank-one recognition rate of the entire asymmetric face recognition system is up to $95.4 \%$ obtained on the entire FRGC v2.0 database, which is better than those of most traditional 2D-2D face recognition methods, and not far behind 3D-3D based ones (range from $96 \%$ to $98 \%$, precise numbers are omitted due to limited space). On the other hand, it avoids the high online cost of data acquisition and computation commonly occurred in 3D-3D face recognition.

\section{Conclusions}

This paper presented an asymmetric 3D-2D face recognition method based on a novel biological vision-based facial representation, namely OGMs. These OGMs simulate the response of the complex neuron to gradient information in a pre-defined neighborhood, and hence can describe local texture changes of 2D faces and local shape variations of 3D face models. Due to its property of being highly distinctive, OGMs improve the results of both matching steps of asymmetric face recognition, i.e. (1) 3D-2D matching using Canonical Correlation Analysis (CCA); (2) 2D-2D matching using LBP histogram based features and Sparse Representation Classifier (SRC). Some comparative experiments were carried out on the complete FRGC v2.0 database, and their results clearly demonstrate the effectiveness of the biological vision-based facial representation and its successful application to asymmetric face recognition.

\section{References}

[1] T. Ahonen, A. Hadid, and M. Pietikinen. Face recognition with local binary patterns. In European Conference on Computer Vision, 2004.

[2] F. R. Al-Osaimi, M. Bennamoun, and A. S. Mian. An expression deformation approach to non-rigid $3 \mathrm{~d}$ face recognition. International Journal of Computer Vision, 81(3):302316, 2009.

[3] N. Alyuz, B. Gookberk, and L. Akarun. Regional registration for expression resistant 3-d face recognition. IEEE Transactions on Information Forensics and Security, 5(3):425-440, 2010.

[4] K. W. Bowyer, K. Chang, and P. J. Flynn. A survey of approaches and challenges in $3 \mathrm{~d}$ and multi-modal $3 \mathrm{~d}+2 \mathrm{~d}$ face recognition. Computer Vision and Image Understanding, 101:1-15, 2006.

[5] S. Edelman, N. Intrator, and T. Poggio. Complex cells and object recognition. http://kybele.psych.cornell.edu/edelman /archive.html, 1997.

[6] T. C. Faltemier, K. W. Bowyer, and P. Flynn. A region ensemble for 3-d face recognition. IEEE Transactions on Information Forensics and Security, 3(1):62-73, 2008. 
[7] D. R. Hardoon, S. Szedmak, and J. Shawe-taylor. Canonical correlation analysis: an overview with application to learning methods. Neural Computation, 16(12):2639-2664, 2004.

[8] D. Huang, M. Ardabilian, Y. Wang, and L. Chen. Asymmetric $3 \mathrm{~d} / 2 \mathrm{~d}$ face recognition based on lbp facial representation and canonical correlation analysis. In International Conference on Image Processing, 2009.

[9] D. Huang, M. Ardabilian, Y. Wang, and L. Chen. Automatic asymmetric $3 \mathrm{~d}-2 \mathrm{~d}$ face recognition. In International Conference on Pattern Recognition, 2010.

[10] D. Huang, M. Ardabilian, Y. Wang, and L. Chen. A novel geometric facial representation based on multi-scale extended local binary patterns. In IEEE International Conference on Automatic Face and Gesture Recognition, 2011.

[11] D. Huang, W. Ben Soltana, M. Ardabilian, Y. Wang, and L. Chen. Textured $3 \mathrm{~d}$ face recognition using biological vision-based facial representation and optimized weighted sum fusion. In IEEE Computer Society Conference on Computer Vision and Pattern Recognition Workshops on Biometrics, 2011.

[12] D. Huang, K. Ouji, M. Ardabilian, Y. Wang, and L. Chen. $3 \mathrm{~d}$ face recognition based on local shape patterns and sparse representation classifier. In International Conference on Multimedia Modeling, 2011.

[13] D. Huang, G. Zhang, M. Ardabilian, Y. Wang, and L. Chen. $3 \mathrm{~d}$ face recognition using distinctiveness enhanced facial representations and local feature hybrid matching. In IEEE International Conference on Biometrics: Theory, Applications, and Systems, 2010.

[14] I. A. Kakadiaris, G. Passalis, G. Toderici, M. N. Murtuza, Y. Lu, N. Karampatziakis, and T. Theoharis. Threedimensional face recognition in the presence of facial expressions: an annotated deformable model approach. IEEE Transactions on Pattern Analysis and Machine Intelligence, 29(4):640-649, 2007.

[15] P. J. Phillips, P. Flynn, T. Scruggs, K. W. Bowyer, J. Chang, K. Hoffman, J. Marques, J. Min, and W. Worek. Overview of the face recognition grand challenge. In IEEE Computer Society Conference on Computer Vision and Pattern Recognition, 2005.

[16] C. Queirolo, L. Silva, O. Bellon, and M. Segundo. 3d face recognition using simulated annealing and the surface interpenetration measure. IEEE Transactions on Pattern Analysis and Machine Intelligence, 32(2):206-219, 2010.

[17] A. Rama, F. Tarres, D. Onofrio, and B. Tubaro. Mixed 2d$3 \mathrm{~d}$ information for pose estimation and face recognition. In International Conference on Acoustics, Speech and Signal Processing, 2006.

[18] D. Riccio and J.-L. Dugelay. Asymmetric 3d/2d processing: a novel approach for face recognition. In International Conference on Image Analysis and Processing, 2005.

[19] A. Scheenstra, A. Ruifrok, and R. C. Veltkamp. A survey of $3 \mathrm{~d}$ face recognition methods. In International Conference on Audio- and Video-Based Biometric Person Authentication, 2005.

[20] L. Spreeuwers. Fast and accurate $3 d$ face recognition using registration to an intrinsic coordinate system and fusion of multiple region classifiers. International Journal of Computer Vision, 93(3):389-414, 2011.

[21] J. Wright, A. Yang, A. Ganesh, S. Sastry, and Y. Ma. Robust face recognition via sparse representation. IEEE Transactions on Pattern Analysis and Machine Intelligence, 31(2):210-227, 2009.

[22] W. Yang, D. Yi, Z. Lei, J. Sang, and S. Li. 2d-3d face matching using cca. In IEEE International Conference on Automatic Face and Gesture Recognition, 2008.

[23] W. Zhao, R. Chellappa, P. J. Phillips, and A. Rosenfeld. Face recognition: a literature survey. ACM Computing Surveys, 35:399-458, 2003. 